\title{
Agôn
}

Revue des arts de la scène

HS 2 | 2014

Mettre en scène le conte

\section{Entretien avec Renaud Rubiano, créateur vidéo}

\section{Renaud Rubiano et Marion Boudier}

\section{(2) OpenEdition}

Journals

Édition électronique

URL : http://journals.openedition.org/agon/3068

DOI : $10.4000 /$ agon.3068

ISSN : 1961-8581

Éditeur

Association Agôn

Référence électronique

Renaud Rubiano et Marion Boudier, "Entretien avec Renaud Rubiano, créateur vidéo », Agôn [En ligne], HS 2 | 2014, mis en ligne le 13 juillet 2014, consulté le 15 septembre 2020. URL : http:// journals.openedition.org/agon/3068

Ce document a été généré automatiquement le 15 septembre 2020

Association Agôn et les auteurs des articles 


\title{
Entretien avec Renaud Rubiano, créateur vidéo
}

\author{
Renaud Rubiano et Marion Boudier
}

La vidéo de Cendrillon a été réalisée par Renaud Rubiano, artiste plasticien et vidéaste, par ailleurs co-fondateur de la plateforme didascalie.net dédiée aux écritures scéniques et inter-medias.

Marion BoudieR - Cendrillon a été ta première collaboration avec Joël Pommerat, comment cela s'est-il passé?

Renaud RUBIANO - J'avais rencontré Eric Soyer, scénographe éclairagiste, trois ans auparavant sur d'autres spectacles, et il a pensé qu'avec Cendrillon ce serait le bon moment pour intégrer l'image dans les créations de Louis Brouillard. Tout est parti de là. Depuis j'ai travaillé avec Joël sur deux autres spectacles. Entre Cendrillon, La Grande et Fabuleuse Histoire du commerce et La Réunification des deux Corées, le travail a été à chaque fois très différent. 
La chanson du prince, acte II scène 7

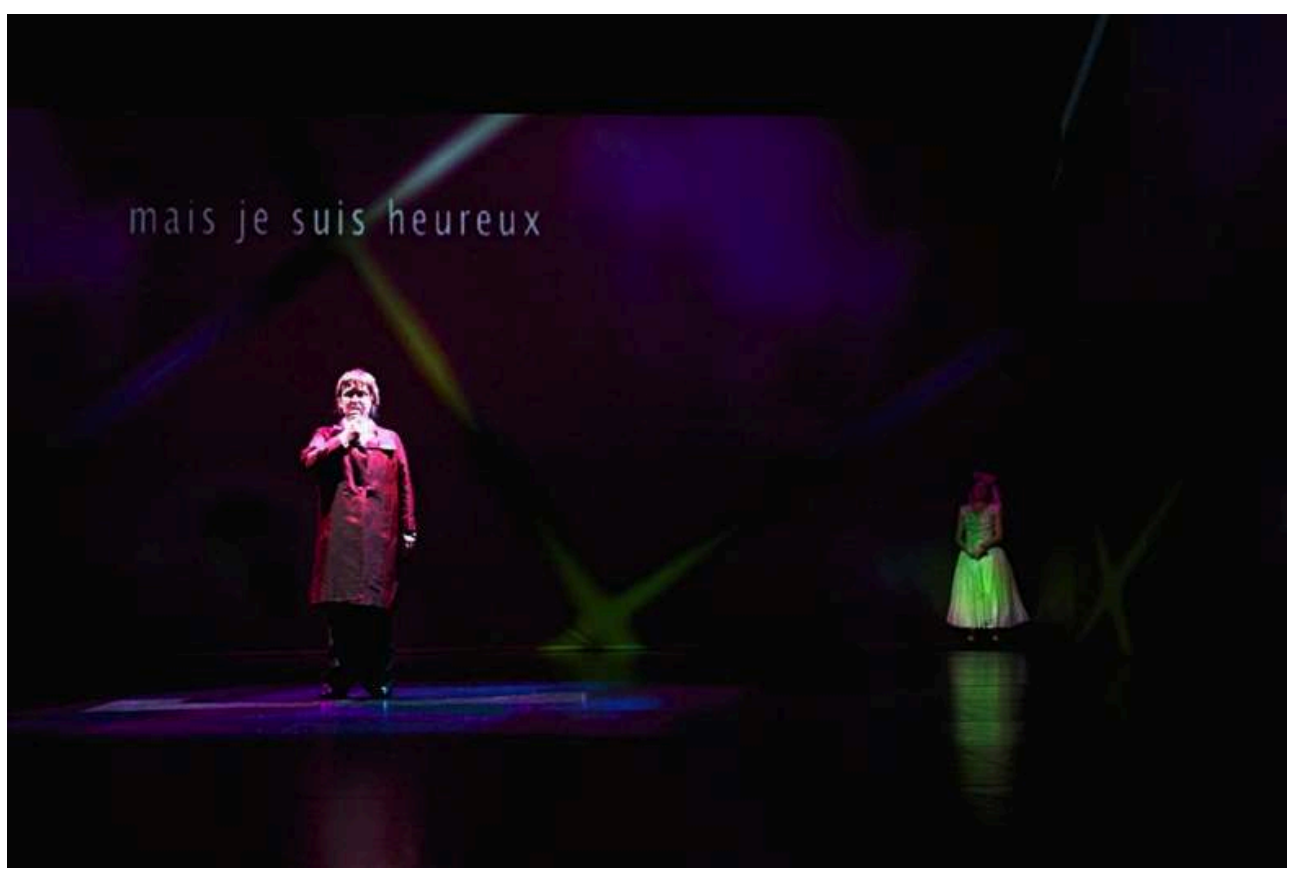

(C) Cici Olsson

M. B. - Les contes me semblent être un espace d'expérimentation particulièrement fécond, par exemple pour le rapport entre le son et l'image dans Le Petit Chaperon rouge, et avec la vidéo dans Cendrillon. En 2006, dans un entretien à la SACD, Joël avait dit vouloir « utiliser la vidéo pour faire évoluer la couleur sur les murs », c'est chose faite avec ton travail dans Cendrillon.

R. B. - Cendrillon a effectivement été l'espace de l'expérimentation vidéo. Cela a démarré au Théâtre National de Bruxelles, où était organisé un stage de deux semaines avec des comédiens dans le but de trouver la distribution. J'ai alors été très libre de proposer des images, des séquences. Nous avions eu un rendez-vous deux mois auparavant pendant lequel Joël m'avait parlé deux heures durant, chez Eric Soyer, et il m'avait confié ses notes sur Cendrillon, une quarantaine de pages dactylographiées, avec toutes ses idées, ses envies, qui préfiguraient sa manière d'aborder le conte, aussi bien dramaturgiquement parlant qu'en terme d'ambiance et d'espace... Cela mentionnait par exemple des couleurs, des brillances, de la transparence. Je m'en suis inspiré pour tourner des images en amont, afin de m'armer de séquences prêtes à diffuser. Ensuite pendant les temps de plateau, je déconstruisais tout cela en temps réel afin de re-construire les images scéniques globales, en étroite collaboration avec Eric.

Durant nos recherches et expérimentations nous avons trouvé des principes vidéos de mouvement qui pouvaient être englobant, voire vertigineux. Nous nous créons au fil du temps une grammaire, une syntaxe que nous distillons ensuite dans les spectacles. Les périodes de recherche de Cendrillon furent très riches, nous n'avons exploité qu'une partie de nos découvertes... On en garde pour la suite !

M. B. - Tu es donc été présent tout au long de la création.

R. B. - Oui, comme chacun dans la compagnie Louis Brouillard (costumes, lumière, son, machinerie). Joël propose une situation aux comédiens, qui vont improviser à partir de ce cadre. Cette action se situe dans un lieu, une maison, une cave, un 
couloir, etc., et à partir de là nous cherchons en lumière et en vidéo de manière conjointe une manière de rendre ce lieu. Lors de la première journée de plateau (mai 2011, durant le stage), Joël m'a demandé d'être libre sur les propositions, ce que j'ai fait. Ensuite, si jamais cela ne lui parlait pas, il demandait une autre proposition, tout en expliquant pourquoi il n'aimait pas celle-ci, mais tout en laissant toujours une grande liberté d'expression. Lors de la phase de travail suivante (septembre 2011) quelques mois après, il a écrit les scènes et il demandait de conserver telle ou telle proposition. Nous lui fournissons des propositions dont il se saisit et qu'il intègre dans son écriture.

Effet de loupe sur le visage de Sandra, acte I scène 3

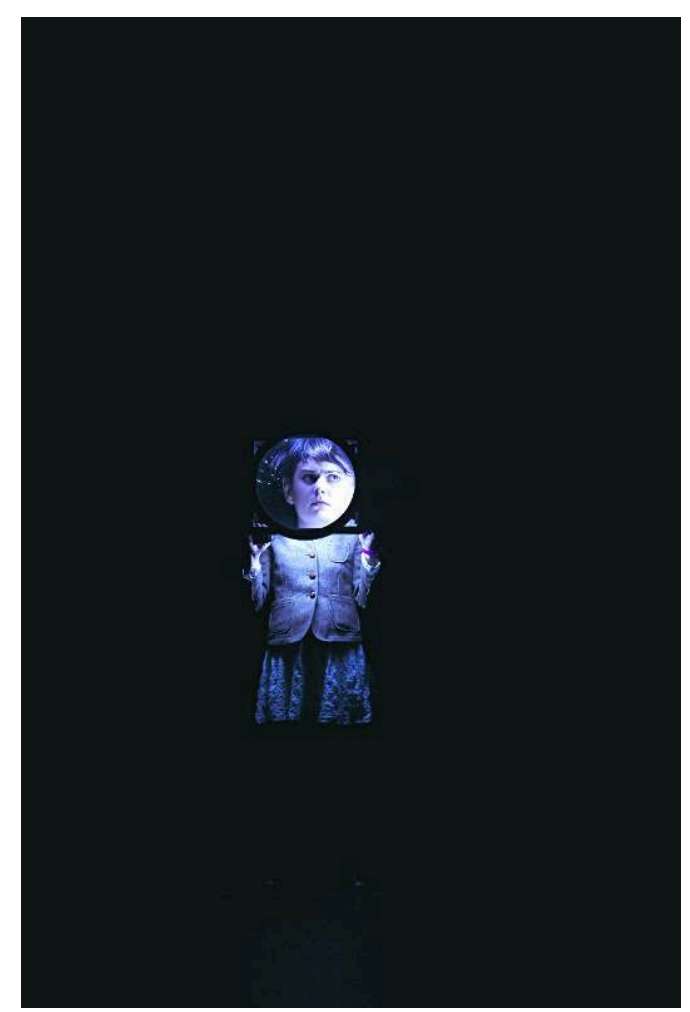

(c) Cici Olsson

M. B. - Peux-tu me parler du lien avec la lumière ? La vidéo étant de la lumière projetée, estce qu'elle peut remplacer l'éclairage ? Comment s'opère le mélange entre vidéo et lumière?

R. B. - C'est exactement le travail que nous avons mené pour La Réunification des deux Corées. La vidéo ne peut pas remplacer complètement l'éclairage, cela demanderait d'une part trop de video-projecteurs, et d'autre part l'image scénique s'en trouverait appauvrie. Mais vidéo-projection et lumière se complètent merveilleusement bien, car ce sont deux qualités de lumière qui ont chacune leurs spécificités. Quand le mélange est réussi, quand l'alchimie opère, il est très difficile de dissocier la projection video de la lumière traditionnelle. Nous avons passé beaucoup de temps à regarder pour comprendre les dosages et les types de sources à utiliser, avec quels angles. Le plus important est de ne pas avoir de préjugés et d'observer attentivement. 
M. B. - La vidéo apporte des effets de lumières et aussi du réalisme, par exemple pour la représentation de la nature derrière les murs de la maison de verre.

R. B. - La video-projecion sur le plateau théâtral doit avant tout être considérée comme une source lumineuse, car c'est ce qu'elle est. Ensuite, le fait de lui apporter un support pour arrêter le faisceau, tels le décor de Cendrillon va matérialiser ses flux lumineux, et ainsi rendre visible leur impact, leur dessin. Le réalisme est très utile tant qu'on l'utilise avec parcimonie et justesse. Deux traits de lumière peuvent parfois suffire à dessiner une pièce, ou à transformer un immense plateau de théâtre en une minuscule pièce. La représentation naturaliste est intéressante pour contextualiser un moment mais doit s'effacer rapidement. Je ne me vois pas du tout représenter un décor réaliste durant tout un spectacle. Mais en revanche distiller des éléments réalistes permet de donner tout son sens à l'abstraction, et également de situer l'action tout en laissant de la place à l'imaginaire du spectateur. C'est fondamental.

M. B. - Quelles sont tes principales sources d'inspiration?

R. B. - Mes influences sont surtout des artistes visuels, car j'ai une formation de plasticien. James Turrel, Olafur Eliasson, Bill Viola ou Romeo Castellucci sont des artistes qui m'ont marqué. Ensuite je trouve l'inspiration dans l'observation du réel, dans le voyage. J'ai la chance de beaucoup voyager et j'ai toujours une caméra avec moi qui me permet de prendre des «notes-vidéo" constamment, puis de les retravailler ensuite dans mon studio.

M. B. - Est-ce que les reflets sont de vrais reflets ou de l'image vidéo (par exemple au début de l'acte II lorsque Sandra lave les vitres sur lesquelles se reflètent ses belles sœurs) et comment est fabriqué l'effet de loupe, qui est devenu l'image emblématique du spectacle? Est-ce que l'idée de représenter la mère en vidéo était présente dès le début de la recherche?

R. B. - C'est une vraie loupe ! C'est une lentille de projecteur qu'Eric a modifiée pour créer cet effet. Les reflets des comédiens dans les vitres sont des reflets naturels, le Plexiglas offrant, comme le verre, cette possibilité. La représentation de la mère était une idée forte de Joël dès le départ, ce qui m'a permis de prendre le temps de peaufiner cette image. Ce point de départ de l'histoire a été travaillé durant plusieurs semaines avec la scène jouée dans les coulisses en live, filmée et projetée sur scène afin de comprendre les enjeux de cette mise en image et d'affiner le jeu. Enfin, nous avons tourné la scène deux semaines avant la première. 
Retour au chevet de sa mère en compagnie de la fée, acte II scène 14

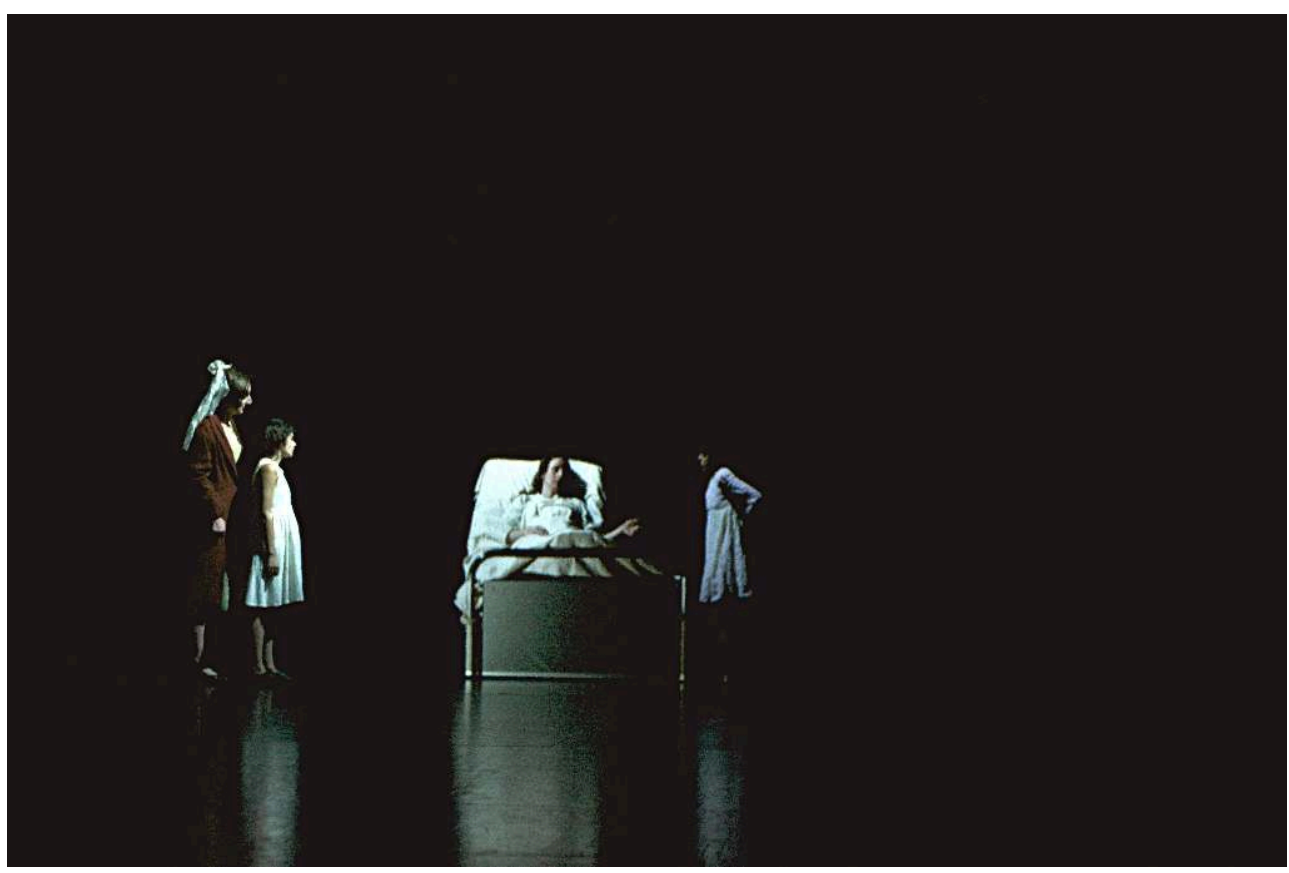

(C) Cici Olsson

M. B. - Tu a fondé un studio dédié aux «écritures vidéo», comment définis-tu cette notion?

R. B. - Je parle d'écritures vidéo, car elles sont multiples suivant leur contexte. Je travaille aussi bien pour le spectacle vivant, que pour des créations d'installations plastiques, parfois aussi pour des projets plus commerciaux tels des show-rooms d'horlogerie ou de joaillerie. C'est pour moi très important, car ce sont autant de manière différente d'aborder la notion d'image. Chaque projet permet d'enrichir l'autre, même s'il n'est pas de la même «famille ». Je m'ennuierais trop à travailler toujours le même type d'images. Et puis cela permet de mélanger les genres, de ne pas s'enfermer dans des codes de représentation qui pourraient brider et enfermer l'imaginaire. C'est tout l'enjeu du Studio Mirio.

M. B. - Cendrillon me semble être un bon exemple d'écrire vidéo en lien avec la musique, la lumière et le corps : y a-t-il une scène dans laquelle ton travail te semble particulièrement abouti (ta scène préférée !) ?

R. B. - Cendrillon est un très bon exemple de ce lien entre la musique, la lumière et le corps. S'il manque un des éléments, les autres perdent leur sens. C'est la base du travail d'équipe pour le spectacle vivant. La maison de verre est pour moi une merveille, car elle mêle pleinement la vidéo et la lumière à tel point qu'on crée un véritable espace en abolissant les frontières du plateau théâtral. Ça n'est pas que mon travail soit plus abouti dans ces scènes, mais c'est un endroit où il y a une vraie cohérence entre les différents média, lumière, vidéo et son. Le théâtre, comme la danse, n'est pas un endroit où l'on doit penser à son travail avant tout, mais à l'ensemble. On peut avoir le plus beau film du monde à projeter, il ne sera pas crédible ou juste s'il n'est pas à sa place à ce moment là. 


\section{Photo de tournage}

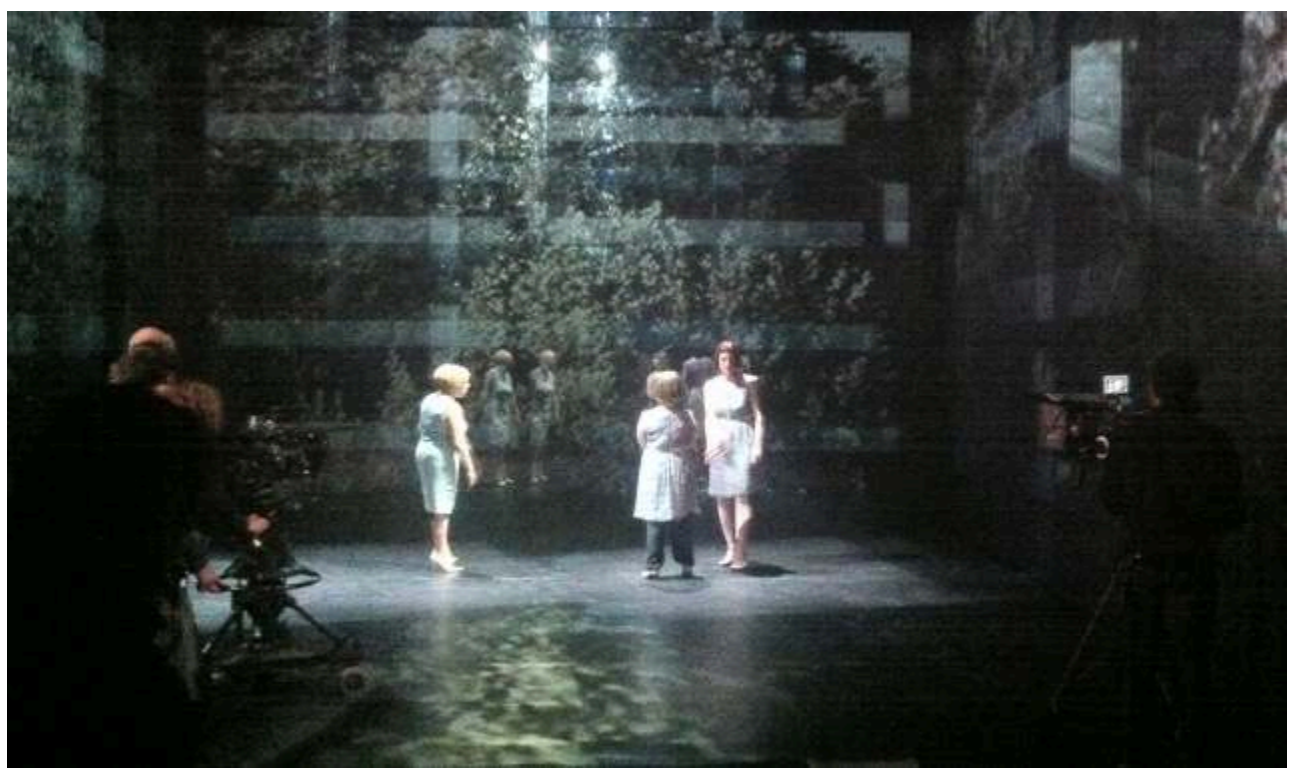

Photo prise pendant le tournage du film du spectacle réalisé par Florent Trochel pour Axe Sud et Arte (c) Renaud Rubiano

\section{INDEX}

Mots-clés : Pommerat (Joël), Rubiano (Renaud), vidéo 